\title{
Una familia de campesinos leoneses a finales del siglo XII
}

\author{
Blas Casado Quintanilla \\ UNED
}

\section{RESUMEN}

Conocemos esta familia a causa de dos contratos de compraventa realizados a finales del siglo xil por los herederos de

Diego, quien a su muerte, deja viuda $y$ siete hijos. Seis herederos, con la autorización de la madre viuda, aunque dispersados por varios lugares, hacen una venta conjunta de su heredad patrimonial al convento de San Marcos de León; el otro heredero, antes excluido, unos meses más tarde, realiza la venta de sus fincas al mismo comprador. El proceso de elaboración de sendos documentos y su análisis comparado dan ocasión a unas reflexiones sobre la vida familiar en aquella sociedad.

\section{PALABRAS CLAVE}

Familia, viuda, labradores, herederos, compraventas, concejos.

\begin{abstract}
We know the existence of this family due to the presence of two sale and purchase contracts carried out at the end of the XIIth century by the heirs of Diego who, when he died, left a widow and seven sons. Six of them carried out a joint sale contract to the convent of Saint Marcus in Leon, with the autorization of their mother widow, although they lived spread among several places. The other heir, who was previously excluded, carried out the sale of his real states to the same purchaser, several months later. The preparation process of both documents and their comparative analysis, give a chance to make several reflections about the family life of that society.
\end{abstract}

\section{KEY WORDS}

Family, widow, country people, heirs, sales and purchases, town councils.

Componen esta familia la viuda de don Diego, cuyo nombre ignoramos, y sus siete hijos. Dos casados: Guterio, casado con doña Toda Nunniz y García, casado con María Munniz'. Cinco solteros: Gonzalo, Fernando, Pedro, Gómez y Rodrigo.

1 Se trata de dos documentos redactados por dos amanuenses distintos. Uno escribe "Tota Nunniz» y el otro "Maria Munniz". No nos atrevemos a afirmar que sean dos formas de escribir un mismo ape- 
Se trata de una familia en la que la única mujer es la madre; cosa un tanto sorprendente, aunque nada podamos indicar acerca de la no existencia de descendencia femenina en la misma. El amanuense de uno y el notario de otro de los documentos que nos sirven de base conceden el tratamiento de "don» al padre y a la mujer de Guterio, el resto de los familiares no reciben ningún tratamiento específico.

Se trata de una familia de pequeños propietarios, que reside en una pequeña población y que salió del anonimato después de la muerte del progenitor. Otras muchas, muchísimas familias de aquella época, no han tenido la fortuna de ser conocidas en la posteridad sino que vivieron y desaparecieron en el más absoluto de los anonimatos: bien porque no tuvieron bienes para legar a sus descendientes, $\mathrm{ni}$ tuvieron voz en su momento; bien porque el documento en el que los consignaron no llegó hasta nosotros; bien porque el amanuense o el escribano que lo consignó no dejó constancia de los acontecimientos con la abundancia de detalles familiares que nos han permitido estas reflexiones. Estamos ante dos documentos que delatan las relaciones de los miembros de una familia entre sí y de su relación con la sociedad en la que les tocó vivir; son personas que participan en un acontecimiento que nos introduce en la sociedad medieval, porque se produjo en la Edad Media, pero que atañe a la familia desde tiempos inmemoriales y que no es especifico de ninguna época.

Por lo general los documentos no dejan constancia de este tipo de situaciones sino que se dedican a cumplir el objetivo contractual para el que fueron concebidos; en cambio son las fuentes literarias las que nos informan no solo de la vida cotidiana, sino también de los acontecimientos que de alguna manera fueron excepcionales o se salieron de lo común. A pesar de ello las fuentes documentales aunque no fueron concebidas para contar detalles de la vida corriente, en algunas ocasiones y con una lectura atenta, muestran aspectos de interés social y ámbito cotidiano $^{2}$, y son reflejo del establecimiento de derechos y obligaciones que atañen a las personas afectadas.

\section{LA PRIMERA VENTA}

A la muerte de don Diego, el padre, nos enteramos que los hermanos Guterio y Garcia, casados, se han quedado en el lugar de residencia de los padres y cul-

Ilido. Si asi fuera, cosa nada descartable, podriamos estar hablando de dos hermanos casados con dos hermanas, lo que sería un elemento de explicación nada despreciable para una parte del tema que nos ha de ocupar a lo largo de estas reflexiones.

2 Prescindimos de citas bibliográficas de los autores que han estudiado la historia de las mentalidades y la vida cotidiana en la Edad Media porque son multitud. Como botón de muestra la "VIII Semana de Estudios Medievales» de Nájera, celebrada en agosto de 1997 y publicada por el Instituto de Estudios Riojanos, Logroño 1998, hay un artículo preparado por Luis Eduardo RODRiguez LATORRE, Pilar SAENz Preciado y Cristina Sigüenza PelaRda en el que se recoge la bibliografía sobre este tema hasta esa fecha, ya lejana, y ocupa nada menos que de la página 407 a 483, y no la consideramos exhaustiva. Más cercano en el tiempo, Maria Luisa Bueno Dominguez, Espacios de vida y muerte en la Edad Media, Edit. Semuret, Salamanca, 2001, aporta un capitulo bibliográfico. 
tivan las fincas del patrimonio familiar, situadas en Quintanilla Seca ó Quintanilla del Olmo, que es un mismo lugar con dos nombres, junto al río Valderaduey, en término de Villalpando, y en Fuente la Piedra; el resto de los hermanos se dispersaron por varios lugares: Perales, Villalba, Villa Samet y León. No parece que los pequeños de la familia tengan la intención de permanecer toda su vida en el lugar de nacimiento y residencia de sus padres o de sus hermanos mayores. Buscan otros lugares de trabajo e incluso otra forma de vida en la ciudad, como es el caso de Rodrigo ${ }^{3}$.

La familia, encabezada por la madre, viuda, deciden vender sus heredades al convento de San Marcos de León en el año 1195; todos aceptan realizar esta venta en enero, mientras que García y su mujer María Muñiz se desvinculan en esta fecha del núcleo familiar y realizan por separado la venta en el mes de abril. Ignoramos, en primer lugar, por qué razón la familia se decide a vender sus fincas patrimoniales al convento de San Marcos, pues los documentos nos niegan este dato; destacamos este extremo porque, al menos, dos de los miembros familiares vivían de la explotación de las citadas fincas y por lo tanto sorprende que ellos mismos tomen parte activa en la venta. Ahora bien, el previsible reparto de la herencia paterna entre todos los herederos traería como consecuencia una parcelación excesiva de las fincas con consecuencias muy negativas no solo para la explotación agrícola, sino también como medio de subsistencia; mantenerlas "pro indiviso" llevaba consigo el pago de una renta a los herederos que no participaban en los beneficios de la explotación agrícola; la compra de sus derechos de propiedad a los hermanos ausentes del lugar y no implicados en el laboreo directo de las fincas, no seria posible, entre otras razones, por falta de medios económicos de Guterio para adquirirlas; venderlas al convento de San Marcos y, posiblemente, seguir con la explotación a cambio de una renta anual, debió parecer a Guterio la mejor de las soluciones. Estas fincas objeto de la compraventa estaban destinadas a tierras de labor o a viñedo, y además tenían un molino en el patrimonio familiar. En el documento no se menciona la casa donde vivían los miembros de esta familia, por tanto no entraba en la venta; lo que hace suponer que Guterio pensaba seguir viviendo alli y continuar con su actividad agrícola.

En el primer documento se indica que se trata de una "venta o cambio" con el prior y convento de San Marcos; en el segundo sólo se habla de una "venta" con el mismo prior y convento. En el primero de los supuestos, el amanuense introduce un elemento de distorsión puesto que se trata de una venta o de un cambio, lo que no es lo mismo; en caso de venta el comprador entrega una cantidad de dinero al vendedor; en el supuesto de que sea venta y cambio, el comprador entrega al vendedor una cantidad de dinero y otras fincas o bienes a cambio, con lo que los vendedores no se quedarían sin tierras para su explotación. La distorsión se deshace si vemos que el comprador se limita a entregar la cantidad de Cll maravedis al vendedor, sin que se haga mención alguna al objeto de un intercambio de

${ }^{3}$ AHN. OO. MM. Santiago. San Marcos de León, carp. 385, núm. 68. 
fincas. En el segundo de los documentos se trata de una venta por la que el vendedor recibe del convento de San Marcos la cantidad de XX maravedís.

El cultivo de las fincas y bienes del patrimonio familiar habia servido para vivir los padres y criar a los hijos. Ahora vemos que, dividido el patrimonio entre los descendientes, no servía o era insuficiente para que todos los hijos pudieran vivir de la explotación del patrimonio familiar. Según parece sólo servía para que dos hermanos pudieran sostenerse siguiendo con la explotación de todo el patrimonio familiar; se trata de dos hermanos casados, esto es, que tenían la posibilidad de añadir a su patrimonio el de sus respectiva mujeres y por esta vía reunir un número de fincas cuya explotación les proporcionara medios suficientes para vivir. Los demás miembros de la familia habían abandonado el solar de origen en busca de mejores perspectivas de vida en otros lugares, habían emigrado rompiendo por esta vía la idea de que el campesino nacía, vivía y moría en el lugar de nacimiento; pero esta dispersión no implicaba perder el derecho sobre el patrimonio familiar, antes al contrario, seguian siendo propietarios de los bienes paternos, en igualdad de condiciones que los demás hermanos. Esto es, las fincas y el molino no estaban repartidas entre los hermanos, aunque cabe suponer que eran explotadas por los herederos que vivian en Quintanilla y Fuente la Piedra.

Las relaciones familiares debían ser francamente mejorables. A primera vista, Guterio y su mujer Toda, juntamente con Gonzalo, lamentamos ignorar los motivos, convencen a la madre, viuda, para hacer una venta de las fincas y repartir el beneficio de la venta entre todos los herederos; también pudiera haber sucedido que la idea de la venta naciera de la madre quien, ante la discordia entre los hermanos, $y$, dada la discordia, la madre hubiera consultado la situación en la que se encontraba con alguna persona de su confianza a la que hubiera demandado algún remedio a una situación poco grata para ella; ¿acaso por esta vía "de solicitud del consejo" se produjo la intervención del convento de San Marcos? La estructura del documento, primero en la cláusula de confirmación y la cualidad de los confirmantes avalan una y otra posibilidad ${ }^{4}$. En uno y otro supuesto, queda de manifiesto que existe la discordia entre los hermanos. De un lado, García y su mujer María, se apartan de la venta realizada en el mes de enero de 1195; y de otro lado, queda clara la situación de desacuerdo porque se busca y se consigue la intervención de los concejos de Quintanilla y de Villalba, con un buen número de testigos en cada caso. Hemos comprobado en otras muchas ocasiones que el recurso y la intervención de los concejos en los contratos de compraventa entre los particulares pone de manifiesto la existencia de diferencias de interés entre los participantes en un contrato de compraventa o la situación de litigio en la que se encuentra el bien objeto de venta; sin que esto excluya un buen número de intervenciones del concejo en la corroboración de un contrato de compraventa como si de un acto establecido por la costumbre y rutinario se tratara, lo que nos

4 Se dice en el primer documento: «in primis ad concessum Guterii Didaci et uxoris eius Tota Nunniz", a los que siguen varios presbíteros en calidad de confirmantes. 
situaría en unas circunstancias bien distintas. En las primeras, el concejo apacigua las disensiones y sirve de garante de la compraventa; en el segundo supuesto, el concejo celebra y ratifica el acuerdo alcanzado entre el comprador y el vendedor ${ }^{5}$.

Lo cierto es que el primer titular de la compraventa es Guterio Díaz con su mujer doña Toda, quien obra, de un lado, en representación de sus hermanos, "excepto Garsia Didaci», y de otro lado, con el consentimiento de su madre. Pero no es menos importante subrayar que otro heredero Gonzalo Díaz, que vive en $\mathrm{Pe}$ rales, se convierte asi mismo en titular del documento y encabeza la lista de los hermanos que con sus propias manos corroboraron y confirmaron la venta a favor de San Marcos.

Parece, por tanto, que son Guterio y su mujer Toda, los que toman la iniciativa de realizar esta venta y son los primeros en firmar la escritura ante unas personas destacadas de Quintanilla Seca como son don Miguel, el capellán, y don Juan Ranna, presbitero. Al mismo tiempo, y, con toda posibilidad porque lo juzgan necesario, acuden nada menos que en busca del apoyo, del otorgamiento y firma de otros muchos confirmantes del concejo de Quintanilla, para disipar toda duda acerca de la validez de esta venta convirtiéndola en pública y notoria en el lugar de residencia de los vendedores. Por último, no contentos con los testigos ordinarios en este tipo de documentos, acude al "concejo de la villa".

En segundo lugar aparece el consentimiento de Gonzalo, que vive en Perales, quien cuenta con el apoyo de la abadesa María Nunniz, más un confirmante de Castellanos de Carrión y otro de San Julián de Saldaña; pero la población de Perales no forma por sí mismo un concejo y por lo tanto no se otorga tal consentimiento concejil.

En tercer lugar suscribe el documento el heredero Fernando Diaz que vive en Villalba, quien además de confirmantes clérigos y laicos, cuenta con el visto bueno y la suscripción del concejo de la villa.

Eri cuarto lugar, Guterio y Gonzalo toman la iniciativa y se desplazan a Villa Samet y aquí recogen la firma de sus hermanos Pedro y Gómez, juntamente con el respaldo de unos cuantos confirmantes, además de la validación del concejo de la villa.

En quinto lugar será en el monasterio de San Marcelo de León donde firme la escritura el último de los herederos que está de acuerdo en la venta. Rodrigo asume y firma la escritura ante el capellán, el abad y un buen número de personas que "uiderunt el audierunt» el acto de aceptación. El redactor del documento deja constancia de su interés, una y otra vez, porque sean muchos los testimonios, de personas destacadas o no, quienes den su conformidad al acto jurídico del que tra-

5 Blas CASAdo Quintanilla, "Pan, vino y documentos de compraventa en León hasta el año 1300", en Escritos dedicados a José María Fernández Catón, Colección Fuentes y Estudios de Historia Leonesa, núm. 100, León, 2004, 163-198. 
tamos. El documento no aporta ningún dato que nos permita conocer las razones por las cuales Rodrigo Díaz realiza la suscripción en el monasterio de San Marcelo de León; pero si hubiera formado parte de la comunidad monástica el amanuense hubiera dejado alguna constancia de esta circunstancia, salvo que al ser nombrado en el último lugar en la lista de los hermanos tengamos que pensar que aún era de corta edad, y todavía estubiera en período de formación en el ámbito eclesiástico.

Se trata de un acto jurídico que ha tenido un largo proceso de elaboración. Desde que se tomó la decisión de realizar la venta hasta que se concretó en un documento escrito ha pasado mucho tiempo. El propio documento nos indica que o bien se ha realizado en varios momentos, o bien se escrituró a base de otros muchos documentos elaborados en los distintos lugares en los que los titulares fueron aceptando el acto jurídico que nos ocupa.

\section{LA SEGUNDA VENTA}

El segundo documento ${ }^{6}$ es mucho más sencillo en todos sus extremos, aunque es inevitable estudiario en su relación con el primero. Ahora, tres meses más tarde, es García Díaz, el miembro de la misma familia que no quiso participar en la venta anterior, quien, en compañía de su esposa María Muñiz, vende al prior y convento de San Marcos una heredad en Quintanilla del Olmo y en Fuente la piedra. No se hace referencia al consentimiento de su madre, como habian hecho sus hermanos. Ante esta circunstancia distinta a la que observamos en la primera venta, caben algunas reflexiones: una, que no lo necesitara, porque su madre no tenían nada que ver en las fincas objeto de esta venta realizada por García; dos, que, con todo, no podemos dejar a un lado la variante jurídica del estado de viuda de la madre y sus posibles relaciones con la herencia patrimonial del esposo difunto; y tres, que su madre se hubiera casado en segundas nupcias o hubiera muerto $^{7}$. En relación con la primera de las posibilidades se nos plantean algunas dificultades. En el primero de los documentos se había consignado que todos los hermanos, con el consentimiento de su madre, querían vender las fincas y bienes heredados de su difunto padre, menos García; esta «excepción nos autoriza a pensar que Garcia tenía su parte en los bienes de su padre, aunque no los queria vender, o que ya no participaba de la propiedad "pro indiviso" con sus hermanos porque él se habia tomado su parte, por reparto o por compra. En ambos supues-

6 AHN. OO. MM. Santiago. San Marcos de León, carp. 385, núm. 69

7 Esta cuestión ha sido objeto de estudio y polémica entre los historiadores del Derecho en la Edad Media Peninsular, porque las fuentes, aunque son muy claras y continuas en algunos aspectos, son discontinuas y admiten muchas variables, en otras ocasiones. No es nuestro objetivo ahora hacer una exposición de este tema, sino que lo traemos a colación por el interés que puede tener en relación con unas compraventas realizadas con la intervención o no de un cónyuge viudo. En su día Martínez Gijón estudió estos aspectos y después los actualizó Enrique GAZTO FERNÁNEZ, La condición jurídica del cónyuge viudo en el derecho visigodo y en los fueros de León y Castilla, Universidad de Sevilla, Sevilla, 1975. 
tos la expresa mención de "excepto García Didaci» hace referencia a una separación real y expresa de García del resto de los hermanos con relación a una propiedad "común", procedente de la herencia paterna. El lenguaje lacónico de la intitulación del documento primero nos permite pensar que García demandó y consiguió su parte en el "pro indiviso" familiar. Esta circunstancia debió causar un disgusto al resto familiar y un quebranto de la salud de su madre.

En el mes de abril de 1195, García y su esposa venden unas fincas al convento de San Marcos en los mismos lugares de Quintanilla y Fuente la Piedra en los que tenian situados sus hermanos los bienes "pro indiviso", lo mismo que el propio García. Todo lleva a pensar que lo que ahora vende García es la parte que debió separarse del "común» hace algún tiempo. La primera venta le costó al convento ciento dos maravedís y esta segunda venta veinte maravedís. El precio de la primera venta hay que dividirla entre seis propietarios, lo que daría como resultado a diecisiete maravedís por cada uno de los vendedores; en la segunda venta el convento le pagó veinte maravedís, lo que significa una ganancia para Garcia. Pero no existe una gran diferencia de valoración, diferencia que podía deberse a una mejor situación de estas fincas o a las mejoras realizadas por García en las mismas, o al interés del convento por comprar esta parte.

Pero por qué razón García se desvincula de sus hermanos en la primera venta y unos meses más tarde realiza la venta de sus heredades al mismo comprador. La presión a la que debió estar sometido, ¿fue personal e individual o debido a la mala situación con sus hermanos, o, por el contrario, fue causada desde el exterior, desde el comprador o desde los convecinos? El documento de la venta es muy lacónico, y nos niega estos datos; en la validación aparecen muchos confirmantes, como es habitual en este tipo documental. Pero destacamos la ausencia de la garantía del concejo, a quien vimos participar en la primera venta, circunstancia y apoyo que en esta ocasión no se juzgó necesario porque la venta se realizó sin que mediaran dificultades especiales o que fueran consideradas así por el vendedor o por el comprador.

\section{EL PROCESO DE ELABORACIÓN DE LOS DOCUMENTOS}

En todo documento hay que distinguir, al menos, dos momentos en el proceso de elaboración: uno, aquel en el que se desarrolla la accićn juridica que va a ser escriturada; otro, aquel en el que la acción jurídica se pone por escrito, esto es, aquel en el que se redacta el documento que ha llegado hasta nosotros. Si tuviéramos que expresarnos en "román paladino" diríamos que una cosa es el documento que leemos y otra bien distinta y anterior es la trastienda que dio origen al documento escrito. La lectura del documento tiene que tener presente aquel proceso previo si quiere ser ampliamente comprensiva.

En casi todos los casos leemos con facilidad el nombre del autor o autores del documento, el del destinatario o destinatarios, y por supuesto, captamos con todo 
detalle el acto jurídico allí consignado, incluso atendemos a la validación documental; ningún otro elemento tiene interés. Solamente en algunas ocasiones encontramos documentos que nos delatan la trastienda que arrastra el proceso de elaboración de los mismos.

El documento del mes de enero de 1195 nos sitúa en este segundo supuesto y nos permite seguir algunos de los pasos dados por los autores antes de llegar a poner por escrito el acto jurídico realizado. El redactor del mismo nos dice que el titular es Guterio, uno de los hermanos, diremos mejor uno de los herederos. Así el amanuense nos dice que Guterio actúa junto con todos sus hermanos, a los que no nombra, mientras que hace expresa mención del excluido de entre los titulares. Nos indica el escribano, por tanto, que hubiera o no una reunión de todos los hermanos-herederos para tratar el tema, extremo este muy dudoso pues no se menciona ni se alude para nada, él conoce muy bien que García queda fuera del acto jurídico documentado, lo que supone una previa reunión de al menos los hermanos Guterio y García.

Se señala a Guterio como primer responsable del acto jurídico y de su consignación e incluye a la madre y viuda, quien dio su consentimiento a la propuesta del conjunto de los herederos de vender las fincas que quedaron de Diego, el padre difunto. A continuación se señala al destinatario o beneficiario de la venta en la persona del prior y de la comunidad de San Marcos, a quien subordina a la Orden de Santiago. El redactor, conocedor del derecho, destaca que el objeto de esta venta son los bienes patrimoniales, señala el precio de la venta, indica el lugar donde se encuentran los bienes, para terminar con la descripción de los mismos. La cláusula en la que enumeran y localizan los bienes objeto de la compraventa ha sido considerada como un formulismo contractual en esta época. A nuestro entender, se puede defender esta idea en tanto en cuanto la cláusula es una parte más de un formulario general que el notario debe «rellenar» en un contrato de compraventa. Es de muy dudosa defensa si se realiza un análisis comparativo del contenido de esta cláusula en distintas series documentales; este estudio da como resultado que el redactor del documento enumera realmente los bienes objeto de la compraventa concreta y que en cada caso se indican bienes distintos porque son distintos los contenidos de una y otra compraventa. La similitud innegable del contenido de esta cláusula en muchos documentos habrá que atribuirla a que, en una economía basada en la agricultura de parcelas de reducida extensión y de pequeños propietarios, todos compran y venden casi los mismos bienes objeto de las explotaciones agrícolas.

Hasta aquí se había mantenido el orden ordinario en que aparecen las cláusulas documentales en este tipo de documentos; en este momento se interrumpe para introducir una cláusula que debiera ir más adelante y en otro contexto. El amanuense sabe que en la escritura tiene que enumerar y nombrar con su apellido al resto de los hermanos-herederos, cosa que no ha hecho en la intitulación donde debió hacerlo, pero a nuestro entender no es un olvido. Allí, en la intitulación, colocó al primer responsable con nombre y apellido y al heredero excluido del contrato que se escritura, señala el carácter patrimonial de los bienes a vender y 
destaca el consentimiento de la madre; ahora, en la corroboración, coloca al resto de los hermanos que están de acuerdo con el primer responsable que es el que actúa en representación general de los hermanos; estos son los que consienten en el acto y por lo tanto deben aparecer con nombre y apellido en la corroboración, porque es lo que sucedió en la realidad, como veremos al analizar la validación documental. Es evidente que el redactor, por un lado, participó en la trastienda del proceso, y por otro, tiene mucho interés en escribir las cosas tal como sucedieron en el tiempo; a la postre es consciente de estar escriturando un documento que ha nacido de forma polémica.

A continuación aparecen las cláusulas penales y después los datos cronológicos y la datación histórica, tras la que comienza la validación que es el elemento documental que despertó nuestra atención y motivó esta reflexión.

En la validación se describe con detalle el largo proceso previo a la venta. Intervienen directamente, como no podía ser de otra manera, los autores del acto jurídico, y participan los testigos y confirmantes que conocieron la trastienda del proceso. Cabe pensar, y aquí aparece el elemento no común de los actos ahora escriturados, que los autores reclamaron la participación del concejo de la villa entre los confirmantes que conocieron y dieron su consentimiento, como garantía de la validez del acto. Pero no solo en la villa de Quintanilla Seca o del Olmo, sino también en Villa Samet y en Villalba, y muchos testigos en San Marcelo de León. El proceso fue largo porque en cada uno de los lugares indicados, a los que hay que añadir el lugar de Perales, se realizó el acto jurídico y la validación del acto jurídico y se tomó nota o se escribió un documento que no ha llegado hasta nosotros, documentos que después se resumieron y se fundieron en la cláusula de validación de uno solo, que es el que nosotros conocemos.

Pero ¿quién de entre los autores reclamó la presencia del concejo o de muchos testigos? ¿Los vendedores o los compradores?. En un análisis comparativo entre el primero y segundo de los documentos nos encontramos con que hay variación entre los autores o vendedores y se trata de objetos de venta distintos, mientras que el comprador es el mismo. Creo que la responsabilidad no debe achacarse, en su caso, sólo a la situación jurídica de los bienes objeto de compraventa, al menos sólo a la situación jurídica de los bienes, pues el redactor deja bien claro el origen del título de propiedad de la heredad de la que se trata, sino a la dispersión de los autores del primer documento. Esto no es obstáculo para que los compradores, por su parte, demanden la mayor seguridad jurídica y la mayor notoriedad posible del acto jurídico, tal como lo exigía el Fuero de León de 1017: "paremus illas scripturas in concilio et inquiramus veritatem, sicut lex docet” ${ }^{8}$. El fuero no hace otra cosa que recoger lo que la ley enseña, y la experiencia

${ }^{8}$ José Manuel Pérez-PRENDES y MUÑOz DE ARRACO, “La potestad legislativa en el reino de León», en El reino de León en la Alta Edad Media, I. Cortes, Concilios y Fueros. Colección Fuentes y Estudios de Historia Leonesa, núm. 48, León, 1988, 497-545. José Antonio FERnÁndez FlóREz, La elaboración de los documentos en los reinos hispánicos occidentales (Siglos vi-XII), Institución Fernán González, Burgos, 2002. 
aconseja, en manifiesta referencia a evitar alteraciones documentales y sus efectos negativos. Pone el foco en salvar la autenticidad documental y la notoriedad de lo documentado y para ello nada mejor que recurrir al concejo, conocedor de la situación, cuya palabra individual al tiempo que colectiva, proporciona una mayor continuidad y publicidad del testimonio, lo que hace muy difícil invalidarlo. En este mismo sentido nos encontramos con que en la cláusula penal de casi todos los documentos de fecha anterior, próxima y posterior a la de los que nos ocupan, se incluye, además de las penas espirituales y pecuniarias, la expresión: "et careat uoce» 0 "et uox eius irrita habeatur». Esta sanción no debe interpretarse como la posibilidad que la ley concede de imponer un castigo físico al infractor, sino como una pena jurídica, esto es, se afirma que su voz sea vana e inútil en el supuesto de reclamación de algún derecho de propiedad sobre el bien objeto de la venta ${ }^{9}$, distinto al que en el documento se determina, máxime si tiene frente a su palabra la de todo el concejo del lugar.

\section{LOS DOCUMENTOS}

1195, enero, 1.

Guterio Díaz y su mujer Toda, juntamente con sus hermanos: Gonzalo, Pedro, Gómez, Fernando y Rodrigo Díaz, excepto García, venden a San Marcos y a la Orden de Santiago una heredad, heredada de su padre, sita en Quintanilla Seca y en Fuente de la Piedra.

A. AHN. OO. MM. Santiago. San Marcos de León, carp. 385, núm 68.

B. AHN. OO. MM. Santiago, TSML, IV, doc. 48.

( Christus) In Dei nomine. Ego Guterius Didaci una cum uxore mea domna Tota et omnibus fratribus / meis, excepto Garsia Didaci, assensu et autoritate matris mee, facimus kartam uendi / cionis et commutacionis priori et conuentui Sancti Marci et ordini Sancti lacobi de omni hereditate / quam habemus uel habere debemus de adquisicione patris nostri domni Didaci pro C II morabetinis in Quintanella Sica l et Fonte de la Penna, scilicet, terris, uineis, molendino, pratis, pascuis, exitu et egressu et omnibus directu / ris suis ut habeant et possideant eam iure hereditario. Et ego Gundisaluus Didaci, similiter cun fratribus meis, / uidelicet, Guterio Didaci, Petro Didaci, Gomez Didaci, Fernando Didaci, Roderico Didaci, hanc kartam quam facere / iussimus propriis manibus roboramus et confirmamus.

Si quis igitur hoc nostrum factum disrumpere presumpserit / omnipotentis iram incurrat, et insuper pectet mille morabetinos.

${ }^{9}$ La expresión usada de manera constante por los amanuenses que han elaborado la documentación referente a San Marcos de León es: “et careat uoce». Pero con datación de 24 de abril de 1125, encontramos este enunciado: "et uox eius pro nichilo reputetur", que se presenta como una fórmula juridica aprendida y repetida en muchas ocasiones. 
Facta karta sub era $\mathrm{M}^{\mathrm{a}} \mathrm{CC}^{\mathrm{a}}$ / $\mathrm{XXX^{ \textrm {a } }}$ III $^{\mathrm{a}}$ et quotum kalendas ianuarii. Regnante rege Adefonso in Legione, Strematura, Gallecia / et Asturiis. Domnus Manricus, episcopus in Legione.

Qui presentes fuerunt:

In primis ad concessum Guterii Didaci et uxoris eius Tote Nunniz. In supradicta uilla:/ domnus Michael, capellanus, conf. Domnus lohannes Ranna, presbiter, conf. Domnus Andreas, conf. Fratres supradicti capellani, conf. Et concilium huius predicte uille, conf.

Ad concessum Gundisalui Didaci, in Perales: abbatissa domna Maria Nunniz, conf. Guterius Petri de Castellanos de Carrion, conf. Nunio Cecus de Sancto luliano de Saldaña, cont.

Ad concessum Fernandi Didaci in Uilla Alua, clerici lohannes Fructuosi. Iohannes, comes, conf. Laici: domnus Andreas, conf. Domnus Lazarus, conf. Dominicus Iuliani, conf. Dominicus Felici, conf. Martinus Felici, conf. Insuper concilium uille, conf.

Postea ad concessum Guterii Didaci, Gundisalui Didaci, Petri Didaci, Gomez Didaci in Uilla Samet, in primis:

( $1^{\mathrm{a}} \mathrm{col}$.) Petrus Fernandi, clericus, conf.- Laici: Iohannes Dominici, conf.- Dominicus Didaci, conf.- Lupus de Lacuna, (signo) conf.-

$\left(2^{a} \mathrm{col}\right.$ ) (signo) Gundisaluus Martini, conf.- Dominicus Fernandi, conf.- Petrus Egidi, conf.- Didacus Saluatori, conf.-

( $\left.3^{a} \mathrm{col}\right)$ Dominicus lohannis Amo, conf.- Dominicus Aprilis, conf.- Iohannes Gallecus, conf.- Petrus Spina, conf.- Insuper et concilium ipsius uille, conf.-

Ad concessum Roderici Didaci in Legione ad Sanctum Marcellum: domnus Isidorus, capellanus Sancti Marcelli, conf. Dominicus, abbas, conf. Dominicus Peronella, conf. Domnus Galter, conf. Domnus Giraldus de Montemirac, conf. Iohannes Baraca, conf. Martinus lohannis, conf. Domnus Rodericus Palliparius, conf. Aparicius, conf. Dominicus, carpentero. Stephanus Agalon. Hii omnes uiderunt et audierunt.

1195, abril, 13.

García Diaz y su mujer Maria Muñiz venden al prior y convento de San Marcos una heredad sita en Quintanilla del Olmo y Fuente de la Piedra.

A. AHN. OO. MM. Santiago. San Marcos de León, carp. 385, núm. 69.

B. AHN. OO. MM. Santiago, TSML, IV, doc. 49.

In Dei nomine. Ego Garsias Didaci una cum uxore mea Maria Muniz facio cartam uenditionis / uobis domno Micaeli priori et conuentui Sancti Marci de omni 
hereditate quam habeo uel habere debeo / in Quintanella de Ulmo et in Fonte de la Petra pro XX morabetinis ut habeatis et possideatis eam iure heredita / rio et de precio apud uos nichil remansit.

Si quis igitur hanc cartam infringere temptauerit, omnipotentis iram / incurrat et pectet centum morabetinos uocem pulsanti.

Facta carta sub era $\mathrm{M}^{\mathrm{a}} \mathrm{CC}^{\mathrm{a}} \mathrm{XXX^{ \textrm {a } }} \|^{\mathrm{a}}$ et quodum / idus aprilis. Regnante rege domno Adefonso in Legione et Gallecia, in Asturiis et Strematura. Domnus Manricus / episcopus in Legione. Fernandus Garsie, maiordomus. Rodericus Ordonii, maiorino regia et tenente turres / Legionis.

Qui presentes fuerunt: Petrus Guidilla et pater eius Petrus Micaelis. Fiel Uelasquez. Pelagius lohannis Toro./

( $1^{2}$ col.) Guterius Munniz, conf.- Petrus Sacco, conf.- Gomez Martiniz, conf.Fernandus Gundissalui, conf.- Petrus Gundissalui, conf.-

( $2^{a} \mathrm{col}$.) Rodericus de Zotes, conf.- Martinus Fernandi, conf.- Domnus Egidius de Becaras, conf.-

( $3^{a} \mathrm{col}$.) Rodericus Fernandi, conf.- Petrus Coco, conf.- Anaa Romanez, conf.( $4^{a}$ col.) Filii F. Baston. Mia Naa. Domnus Uelascus.

$\left(5^{a} \mathrm{col}\right.$.) Petrus, ts.- Dominicus, ts.- lohannes, ts.-

De Malua: Martinus Gundissalui, presbiter, conf. Petrus Martini, filius eius, conf.

Isidorus notuit. (signo) 